\title{
Undiagnosed coeliac disease and risk of autoimmune disorders in subjects with Type I diabetes mellitus
}

\author{
T. Not ${ }^{1}$, A. Tommasini ${ }^{1}$, G. Tonini ${ }^{1}$, E. Buratti ${ }^{2}$, M.Pocecco ${ }^{1}$, C. Tortul ${ }^{3}$, M. Valussi ${ }^{3}$, G. Crichiutti ${ }^{4}$, I. Berti ${ }^{1}$, \\ C. Trevisiol ${ }^{1}$, E. Azzoni ${ }^{1}$, E. Neri ${ }^{1}$, G. Torre ${ }^{1}$, S. Martelossi ${ }^{1}$, M.Soban ${ }^{1}$, A. Lenhardt ${ }^{1}$, L. Cattin ${ }^{2}$, A. Ventura ${ }^{1}$ \\ ${ }^{1}$ Department of Reproductive and Development Science, I. R. C. C.S. "Burlo Garofolo" University of Trieste, Italy \\ ${ }^{2}$ Department of Clinical Science, University of Trieste, Italy \\ ${ }^{3}$ Diabetes Service, City Hospital of Monfalcone, Italy \\ ${ }^{4}$ Department of Paediatrics, D.P. M. S. C., University of Udine, Italy
}

\section{Abstract}

Aims/hypothesis. We tested the hypothesis that silent coeliac disease is more frequent than expected in both patients with Type I (insulin-dependent) diabetes mellitus and their first-degree relatives. We evaluated how the presence of other autoimmune disorders in diabetic patients and their first-degree relatives is related to silent, unrecognized coeliac disease. Methods. Sera from 491 subjects with Type I diabetes, 824 relatives and 4000 healthy control subjects were screened for anti-endomysial antibodies and all those subjects who tested positive for anti-endomysial antibodies underwent intestinal biopsy.

Results. We found that the prevalence of coeliac disease was $5.7 \%$ among the diabetic patients and $1.9 \%$ among the relatives, values significantly higher than those found among the control subjects $(p<0.0001 ; p<0.001)$. The prevalence of autoimmune disorders in diabetic patients with coeliac disease was significantly higher than in subjects with Type I diabetes alone $(p<0.0001)$. The prevalence of autoimmune disorders in the relatives with coeliac disease was significantly higher than in those who tested negative for anti-endomysial antibodies $(p=0.01)$.

Conclusion/interpretation. This report provides further confirmation of the high prevalence of undiagnosed coeliac disease among diabetic patients and their relatives. This interesting new finding is the increased presence of other autoimmune diseases in these patients, as well as in their relatives with a delayed diagnosis for coeliac disease. Patients newly diagnosed with coeliac disease showed excellent compliance with the gluten-free diet. This should encourage policymakers to consider introducing an easy-touse screening programme for diabetic patients and their relatives into everyday clinical practice, in order to prevent coeliac-associated symptoms and the onset of additional, more serious auto-immune disorders. [Diabetologia (2001) 44: 151-155]

Keywords Autoimmune diseases, silent coeliac disease.
Autoimmune diseases are known to be clustered not only in the same person but also in different members of the same family $[1,2]$. An increased prevalence of autoimmune disorders has been found both in patients with Type I (insulin-dependent) diabetes melli-

Received: 14 July 2000 and in revised form: 6 October 2000

Corresponding author: T. Not, MD, Clinica Pediatrica, Istituto per l'Infanzia IRCCS "Burlo Garofolo", Via dell'Istria 65/1, 34100 Trieste, Italy

Abbreviations: AEA, Anti-endomisyum antibody; CD, coeliac disease; FDR, first-degree relatives. tus and in their first-degree relatives (FDR) [3, 4]. Recent data support the contention that coeliac disease is an autoimmune disorder triggered by the ingestion of gluten in genetically susceptible subjects $[5,6]$. The association between Type I diabetes and coeliac disease has been recognised for some years [7] and coeliac disease specific immunologic markers used for screening large numbers of patients with Type I diabetes showed that the prevalence of coeliac disease among children and adults with diabetes is higher than suspected, with rates of 2 to $8 \%[8,9]$. It is not clear whether the association between these two autoimmune diseases is related to a common ge- 
netic background only $[8,10,11]$ or, as has recently been proposed, to the fact that long-term exposure to gluten could induce Type I diabetes and other autoimmune disorders in genetically predisposed subjects [12]. Most cases of coeliac disease associated with Type I diabetes do not present gastro-intestinal symptoms, despite the presence of the characteristic intestinal lesions and these cases would be missed without a screening programme [13]. Therefore, onset of Type I diabetes would seem to occur more often when coeliac disease is present but not recognized and consequently not treated [14].

In this paper, we tested the hypothesis that silent coeliac disease is more frequent in both patients with Type I diabetes and their FDR than expected. Moreover, we evaluated how other autoimmune disorders in patients with Type I diabetes and their FDR is related to silent, unrecognized coeliac disease.

\section{Subjects and methods}

Patients and Control subjects. Of the 500 consecutive patients with Type I diabetes referred to three diabetes units (Paediatrics Department of "Burlo Garofolo" Children's Hospital; General City Hospital of Monfalcone; Department of Medicine, University of Trieste) from June 1998 to January 2000, $491(98 \%$ ) (245 men, 246 women, median age 29 years at the time of the study; range 2-70 years; median age at onset of diabetes 15 years; range $2-40$ years; median duration of diabetes 16 years, range 4 months-34 years) agreed to undergo serological screening for coeliac disease by anti-endomysium antibody test (AEA). These 491 patients (Group A) came from 487 different families. In four of these families two members had diabetes.

We also offered 1420 of their first-degree relatives (Group B: 830 parents; 380 siblings; 210 offspring) the opportunity of being screened for coeliac disease. All the patients in group A and all the subjects in group B who agreed to be tested were encouraged to fill out a questionnaire on their personal medical history with the help of a doctor, giving details about autoimmune diseases. The information collected was verified by analysing medical records or by clinical examinations. The healthy control group consisted of 4000 blood donors (2980 men, 1020 women; median age 35 years, range 18-65).

All AEA-positive subjects underwent intestinal biopsy to confirm the diagnosis, following the criteria set down by the European Society for Gastroenterology Hepatology and $\mathrm{Nu}$ trition [15]. A detailed explanation of the study was given to, and informed consent was obtained from, each subject before testing for coeliac disease antibodies. The investigation was approved by the local ethics committee.

Anti-endomysium antibody assay. Serum IgA anti-endomysium antibody (AEA) was measured by means of indirect immunofluorescence assay, using cryostat sections of human umbilical cord tissue, as described previously [16]. Briefly, the sections were incubated for 30 min with the subject's serum diluted 1:5. After washing, sections were incubated for $30 \mathrm{~min}$ with fluorescein-labeled goat anti-human IgA antibodies. The slides were washed and examined using fluorescent microscopy.
Statistical analysis. A two-tailed Fisher exact test was carried out to determine the significance of the differences in the categories which were defined according to the absence or presence of findings. A value of $p<0.05$ was considered significant.

\section{Results}

Prevalence of silent coeliac disease in patients with Type I diabetes and their first-degree relatives. Of the 1420 first-degree relatives approached, 824 (58\%) agreed to be screened for coeliac disease. Among these were 449 parents ( 238 fathers, 211 mothers, median age 52 years, range 25-77); 234 siblings (112 brothers, 122 sisters, median age 27 years, range 5-54) and 141 offspring (65 sons, 76 daughters, median age 15 years, range $3-35$ ). These were all relatives belonging to the 487 families involved. In 246 families, 1 family member was screened, in 154 families, 2 members were screened, in 80 families, 3 members, in 6 families, 4 members and in 1 family, 6 members.

Anti-endomysium antibodies were detected in 28 of the 491 Type I diabetic patients $(5.7 \%, 14$ women, 14 men, median age 25 years, range 3-60 years), in 16 out of 824 first-degree relatives $(1.9 \%, 11$ women, 5 men, median age 23 years, range $3-65$ years) and in 10 out of 4000 blood donors $(0.25 \%, 2$ women, 8 men, median age 40 years, range $28-60$ years). (Type I diabetes subjects vs control subjects $p<0.0001$; FDR vs control subjects $p<0.001$; Type I diabetes vs FDR $p=0.0003)$. The AEA test results were not age-dependent.

Overall, 54 AEA-positive subjects were detected and all underwent small-bowel biopsies, which were classified according to Marsh's modified classification [17]. Diagnosis of coeliac disease was confirmed in all 54 subjects. Intestinal specimens showed total villous flattening (type $3 \mathrm{c}$ ) in 16 biopsies, marked villous flattening (type $3 \mathrm{~b}$ ) in 25 biopsies and mild villous flattening (type $3 \mathrm{a}$ ) in 13 biopsies.

In the patients with Type I diabetes, coeliac disease was diagnosed at the same time as Type I diabetes in 3 subjects (aged 3, 9 and 11 years) and after Type I diabetes in the other 25 (median age at diagnosis of diabetes was 11 years, at diagnosis of coeliac disease, 25 years).

In the first-degree relatives of patients with Type I diabetes and coeliac disease, the prevalence of silent coeliac disease ( 4 of $75,5.3 \%$ ) was higher than in the first-degree relatives of patients with Type I diabetes who tested negative for AEA (12 of 627, $1.9 \%)$, although the difference was not statistically significant $(p=0.08)$.

Multiple autoimmune disorders in Type I diabetes patients with and without coeliac disease. Ten (35.7\%) of the 28 coeliacs patients with Type I diabetes had at least one other autoimmune disease; four of these 


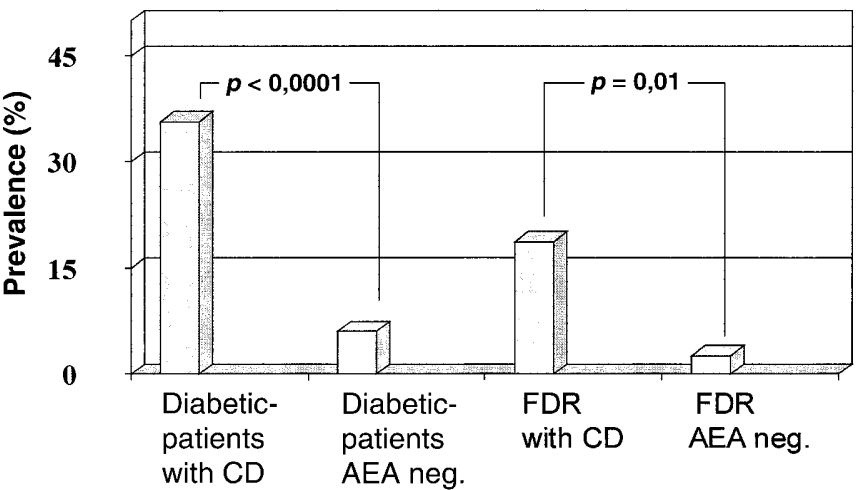

Fig.1. Prevalence of autoimmune diseases in both subjects with coeliac disease (CD) and in subjects tested negative for anti-endomysium antibodies (AEA neg)

were suffering from two other autoimmune diseases. There were three patients with Hashimoto's thyroiditis, two with dermatitis herpetiformis and one with psoriasis. The four subjects with two other autoimmune diseases comprised: one patient with Hashimoto's thyroiditis and autoimmune hepatitis, one patient with Hashimoto's thyroiditis and dermatitis herpetiformis, one patient with Hashimoto's thyroiditis and psoriasis and one with juvenile rheumatoid arthritis and psoriasis.

Of the 463 diabetic subjects testing negative for AEA, 173 subjects filled out the questionnaires $(37 \%)$. Eleven $(6.3 \%)$ of them (11 of 173$)$ had other autoimmune diseases (one patient with Hashimoto's thyroiditis plus vitiligo, four with Hashimoto's thyroiditis, five with psoriasis and one with alopecia areata). The prevalence of autoimmune diseases in patients with both coeliac disease and Type I diabetes was significantly higher than in patients with Type I diabetes alone ( $35.7 \%$ vs $6.3 \%, p<0.0001$ ) (Fig. 1 ).

Three $(18.7 \%)$ of the 16 FDR subjects with coeliac disease had autoimmune diseases (one case of Hashimoto's thyroiditis, one of multiple sclerosis and one of autoimmune hypoparathyroidism).

Of the 808 FDR subjects who tested negative for AEA, 344 subjects filled out the questionnaires $(42.5 \%)$. Nine $(2.6 \%)$ of them (9 of 344$)$ had autoimmune diseases (six cases of Hashimoto's thyroiditis, one of multiple sclerosis, one of psoriasis and one of Hashimoto's thyroiditis and psoriasis). The prevalence of autoimmune diseases in the FDR subjects with coeliac disease was significantly higher than in those testing negative for AEA $(18.7 \%$ vs $2.6 \%$ $p=0.01$ ) (Fig. 1).

Clinical history in coeliac patients discovered by the screening programme. Out of 54 subjects diagnosed with coeliac disease, only $14(26 \%)$ had signs or symptoms that could have been related to gluten intolerance in their clinical history. Seven were Type I diabetes patients (two with recurrent aphthous ul- cers, one with recurrent abdominal pain and diarrhoea, one with sideropenic anaemia, one with hypertransaminasemia, one with dental enamel defect, one with failure to thrive in infancy); four were FDR subjects (one with diarrhoea, one with recurrent abdominal pain, one with failure to thrive, one with diarrhoea, unexplained anaemia and autoimmune hypoparathyroidism); three were blood donors (one with explosive diarrhoea, one with sideropenic anaemia, one with failure to thrive plus diarrhoea in infancy).

All 54 subjects diagnosed with coeliac disease had been on a gluten-free diet and had been followed for at least 12 months $(12-30 \mathrm{~m}$, mean $17 \mathrm{~m})$. At the last visit, $45(83 \%)$ of the 54 coeliac subjects tested negative for AEA. Symptoms and signs that could have been related to gluten had disappeared in eight subjects and haematochemical parameters returned to normal ranges in two subjects with iron deficiency anaemia and in one with hypertransaminasemia.

The gluten-free diet improved the clinical course of autoimmune hypoparathyroidism in a newly diagnosed coeliac patient but the need for pharmacological treatment remained unchanged, as did the degree of clinical control of the other autoimmune diseases (Type I diabetes, Hashimoto's thyroiditis, multiple sclerosis).

\section{Discussion}

It is well known that the prevalence of coeliac disease among patients with Type I diabetes is higher than expected, with rates of 2 to $8 \%[8,9]$. The present findings are in line with other epidemiological studies and show that the prevalence of coeliac disease is significantly greater in diabetic patients $(5.7 \%)$ than in both first-degree relatives $(1.9 \%)$ and healthy control subjects $(0.25 \%)$. Furthermore, the prevalence of coeliac disease among Type I diabetic patients could increase in the long run, because a small percentage of those who initially tested negative could become antibody positive [18]. In view of these observations, it is to be recommended that subjects with autoimmune disease be screened for coeliac disease and that repeat serological screening be carried out about every three years to detect cases of clinically silent, late-onset coeliac disease.

As reported by previous studies $[19,20]$, we observed an increased prevalence of coeliac disease among the first-degree relatives of diabetic subjects. We identified a high prevalence $(1.9 \%)$ of coeliac disease in those families in which the proband was diabetic but not coeliac at the time of the study. This finding suggests that, like other autoimmune diseases, coeliac disease also tends to cluster in first-degree relatives of patients with autoimmune disorders even in the absence of coeliac disease and that antibody 
screening is therefore advisable in these at-risk subjects.

The increased prevalence of autoimmune diseases among subjects with coeliac disease has been welldocumented. Epidemiological data indicates that in almost all those patients who have both diseases, silent coeliac disease was recognised and treated only after diagnosis of the other autoimmune disease $[9$, 21-23].

The frequent association of coeliac disease with autoimmune diseases has usually been explained by a common genetic determinant. Coeliac disease and Type I diabetes share susceptibility alleles in the HLA class II region on chromosome 6 with increased frequencies in both diseases of HLA-DR3 or its associated $\mathrm{DQ} \alpha / \beta$ dimer 0501/0201. In addition, a similar autoimmune pathogenetic mechanism has been shown to be responsible for the development of both diseases, given the presence of organ-specific autoantibodies [6, 24], of T-lymphocyte infiltration at the site of the lesion $[25,26]$, and the etiological importance of environmental risk co-factors [25-27]. Although the possibility of other common susceptibility genes cannot be completely excluded, the influence of environmental factors must obviously be taken into account.

Clear evidence exists of gluten-related autoimmunity in genetically predisposed subjects. It has recently been shown that autoimmune disorders involving organs other than the intestine could develop in unrecognized and/or untreated coeliac subjects because of a persistent exposure to gluten [12].

In this study, the prevalence of autoimmune diseases was found to be significantly greater in relatives with coeliac disease $(18.7 \%)$ than in relatives testing negative for AEA (2.6\%). Moreover, the risk of another autoimmune diseases was higher in Type I diabetes patients with coeliac disease $(35.7 \%)$ than in Type I diabetes patients testing negative for AEA $(6 \%)$. Similar findings were described in our previous study [28] on the relation between coeliac disease and the risk of autoimmune disorders in patients with autoimmune thyroiditis and coeliac disease.

When on a diet containing gluten, some coeliac patients produced organ-specific autoantibodies (GAD, ICA, TPO, anti-Purkinje cells) which were not coeliac-related and which disappeared after 6 to 12 months of a gluten-free diet. It cannot therefore be ruled out that in these subjects, the humoral expression of specific autoimmunity was affected by gluten exposure $[29,30]$.

Bearing these data in mind, an early diagnosis of coeliac disease might have prevented some of our coeliac disease patients from developing an autoimmune disease such as diabetes or thyroiditis. In fact, certain subjects with autoimmune diseases who were diagnosed with coeliac disease by screening showed a relative improvement in the associated disease when placed on a gluten-free diet $[22,31,32]$. No similar phenomenon was observed in our patients with Type I diabetes, autoimmune thyroiditis or multiple sclerosis, probably because of differences in the reparative capacity and functioning of human tissue and also in the duration of the gluten-dependent immune aggression.

Gliadin is an excellent substrate for the tissue transglutaminase, the auto-antigen recognized by AEA [6]. This enzyme cross-links not only gliadin but also several other proteins [33] to make macromolecular complexes causing the formation of new antigens which are both self-epitopes and gliadin epitopes, leading to the production of anti-transglutaminase antibodies and other autoantibodies [34].

It has been suggested that enhanced immune responsiveness to both dietary protein and self-antigens in coeliac disease reflects a failure of immune tolerance in the gut immune system. This is in line with our preliminary studies in which we were able to isolate anti-transglutaminase single-chain antibodies from a phage library derived from intestinal lymphocytes but not from peripheral blood lymphocytes of untreated coeliac disease patients [35]. This means that these auto-antibodies are preliminarily synthesized at the intestinal level and that the failure to achieve tolerance to the autoantigen derives from the gut.

There is evidence to support this assumption in the development of autoimmunity in Type I diabetes. In patients with newly diagnosed Type I diabetes, the islet cell antigen GAD reactive lymphocytes show the gut-specific homing receptors $\alpha 4 \beta 7$ integrin, suggesting that these autoreactive lymphocytes originate in the intestine and also that tolerance to a self-antigen is broken in the gut-associated lymphocyte population [36].

In conclusion, this study confirms that the prevalence of coeliac disease is greater in patients with Type I diabetes than in healthy people. It shows, for the first time that first-degree relatives of Type I diabetes patients constitute a new risk group for coeliac disease. The higher prevalence of other autoimmune diseases in subjects with both coeliac disease and Type I diabetes could be a consequence of the delayed diagnosis of coeliac disease. Diabetic patients would certainly benefit from screening for coeliac disease to eliminate symptoms and limit the risk of developing more severe autoimmune diseases. Our study also shows that compliance to diet in screening-diagnosed coeliac subjects is very high ( $83 \%)$, a further argument for using a screening programme as a preventive measure.

Acknowledgements. This work was supported by grant $46 / 98$ from the I.R.C.C.S. "Burlo Garofolo" Trieste, Italy. We would like to thank the nursing staff: Mrs. S. Ferrara, Mrs. F. Balsemin and Mrs. E. Filaferro for clinical care of the patients. 


\section{References}

1. Neufeld M, Blizzard R (1980) Polyglandular autoimmune diseases. In: Pinchera A, Doniach D, Fenzi G, Baschieri L (eds) Symposium on Autoimmune Aspects of Endocrine Disorders. Academic Press, New York, pp 357-365

2. Lin J, Cash J, Doyle S et al. (1998) Familial clustering of rheumatoid arthritis with other autoimmune diseases. Human Genet 103: 475-482

3. Bottazzo GF, Mann J, Thorogood M, Baum J, Doniach D (1978) Autoimmunity in juvenile diabetics and their families. B M J 2: 165-168

4. Betterle C, Zanette F, Pedini B, et al. (1984) Clinical and subclinical organ-specific autoimmune manifestations in Type I (insulin-dependent) diabetic patients and their first-degree relatives. Diabetologia 26: 341-346

5. Picarelli A, Maiuri L, Frate A, Greco M, Auricchio S, Londei M (1996) Production of antiendomysial antibodies after in-vitro gliadin challenge of small intestine biopsy samples from patients with coeliac diease. Lancet 348: 1065-1067

6. Dieterich W, Ehnis T, Bauer M et al.( 1997) Identification of tissue transglutaminase as the autoantigen of coeliac disease. Nat Med 3: 797-801

7. Savilahti E, Simell O, Koskmies S, Rilva A, Akerblom H (1986) Celiac disease in insulin-dependent diabetes mellitus. J Pediatr 108: 690-693

8. Barera G, Bianchi C, Calisti L et al. (1991) Screening of diabetic children for coeliac disease with antigliadin antibodies and HLA typing. Arch Dis Child 66: 491-494

9. Cronin C, Shanahan F (1997) Insulin-dependent diabetes mellitus and coeliac disease. Lancet 349: 1096-1097

10. Todd J (1990) Genetic control of autoimmunity in Type I diabetes. Immunol Today 11: 122-128

11. Sollid L, Thorsby E (1993) HLA susceptibility genes in celiac disease: genetic mapping and role in pathogenesis. Gastroenterology 105: 910-922

12. Ventura A, Magazzù G, Greco L (1999) Duration of exposure to gluten and risk for autoimmune disorders in patients with celiac disease. Gastroenterology 117: 297-303

13. Vitoria J, Castano L, Rica I, Bilbao R, Arrieta A, GarciaMasdevall M (1998) Association of insulin-dependent diabetes mellitus and celiac disease: a study based on serologic markers. J Pediatr Gastroenterol Nutr 27: 47-52

14. Pocecco M, Ventura A (1995) Coeliac disease and insulindependent diabetes mellitus: a causal association? Acta Paediatr 84: 1432-1433

15. Walker-Smith J, Guandalini S, Schimitz J, Shmerling D, Visakorpi J (1990) Report of working group of European Society for Pediatric Gastroenterology and Nutrition: revised criteria for diagnosis of coeliac disease. Arch Dis Child 65: 909-911

16. Not T, Città A, Lucchesi A, Torre G, Martelossi S, Ventura A (1997) Anti-endomisium antibody on human umbilical cord vein tissue: an inexpansive and sensitive diagnostic tool for the screening of coeliac disease. Eur J Pediatr 156: 616-618

17. Oberhuber G, Granditsch G, Vogelsang H (1999) The histopathology of coeliac disease: time for a standardized report scheme for pathologist. Eur J Gastroenterol Hepatol 11: 1185-1194

18. Maki M, Huupponen T, Holm K, Hallstrom O (1995) Seroconversion of reticulin autoantibodies predicts coeliac disease in insulin dependent diabetes mellitus. Gut 36: 239-242
19. Boudraa G, Hachelaf W, Benbouabdellah M, Belkadi M, Benmansour F, Touhami M (1996) Prevalence of coeliac disease in diabetic children and their first-degree relatives in West Algeria: screening with serological markers. Acta Paediatr Suppl 412: 58-60

20. Hummel M, Bonifacio E, Stern M, Dittler J, Schimmel A, Ziegler A (2000) Development of celiac disease-associated antibodies in offspring of parents with Type I diabetes. Diabetologia 43: 1005-1011

21. Lepore L, Martelossi S, Pennesi M. et al. (1996) Prevalence of celiac disease in patients with juvenile chronic arthritis. J Pediatr 129: 1311-1313

22. Corazza G, Andreani M, Venturo N, Bernardi M, Tosti A, Gasbarrini G (1995) Celiac disease and alopecia areata: report of a new association. Gastroenterology 109: 1333-1337

23. Collin P, Salmi J, Hallstrom O, Reunala T, Pasternack A (1994) Autoimmune thyroid disorders and coeliac disease. Eur J Endocrinol 130: 137-140

24. Bonifacio E, Bingley P, Shattock M et al. (1990) Quantification of islet cell antibodies and prediction of insulin-dependent diabetes. Lancet 335: 147-149

25. Vaarala O (1999) Gut and induction of immune tolerance in Type I diabetes Diabetes Metab Res Rev 15: 353-361

26. Marsh M (1992) Gluten, major histocompatibility complex, and the small intestine: a molecular and immunobiologic approach to the spectrum of gluten-sensitivity ("celiac sprue") Gastroenterology 102: 330-354

27. Richter W, Mertens T, Schoel B et al. (1994) Sequence homology of the diabetes-associated autoantigen glutamate decarboxylase with coxsackie B4-2C protein and heat shock protein 60 mediates no molecular mimicry of autoantibodies. J Exp Med 180: 721-726

28. Berti I, Trevisiol C, Tommasini A et al. (2000) Usefulness of screening program for celiac disease in autoimmune thyroiditis. Dig Dis Sci 45: 403-406

29. Ventura A, Neri E, Ughi C, Leopaldi A, Città A, Not T (2000) Gluten-dependent diabetes-related and thyroid-related autoantibodies in patients with celiac disease. J Pediatr 137: 263-265

30. Hadijvassiliou M, Grunewald R, Davies-Jones G et al. (1998) Gluten ataxia. In: S Lohiniemi, P Collin, M Maki (eds) Changing Features of Coeliac Disease. The Finnish Coeliac Society, Tampere, p 121

31. Brazier F, Delcenserie R, Sevestre H, Delamarre J, Capron J (1994) Primary sclerosing cholangistis and coeliac disease: beneficial effect of gluten free diet on the liver: Eur J Gastroenterol Hepatol 6: 183-186

32. Hadijvassiliou M, Gibson A, Davies-Jones G, Lobo A, Stephenson T, Milford-Wars A (1996) Does cryptic gluten sensitivity play a part in neurological illnes? Lancet 347: 369-371

33. Kommandor E, Achyuthan R, Goodell J et al. (1995) Immunochemical analyses of human plasma fibronectin-cytosolic transglutaminase interactions. J Immunol Methods 180: 69-79

34. Sollid L, Molberg O, MacAdam S, Lundin K (1997) Autoantibodies in coeliac disease: tissue transglutaminase-guilt by association? Gut 41: 851-852

35. Sblattero D, Not T, Marzari R et al. (1999) Phage antibody library from celiac disease patient. J Pediatr Gastroenterol Nutr 28: 568 (Abstract)

36. Paronen J, Klemetti P, Kantele J et al. (1997) Glutamate decarboxylase-reactive peripheral blood lymphocytes from patients with Type I diabetes express gut-specific homing receptor $\alpha 4 \beta 7$-integrin. Diabetes 46: 583-588 